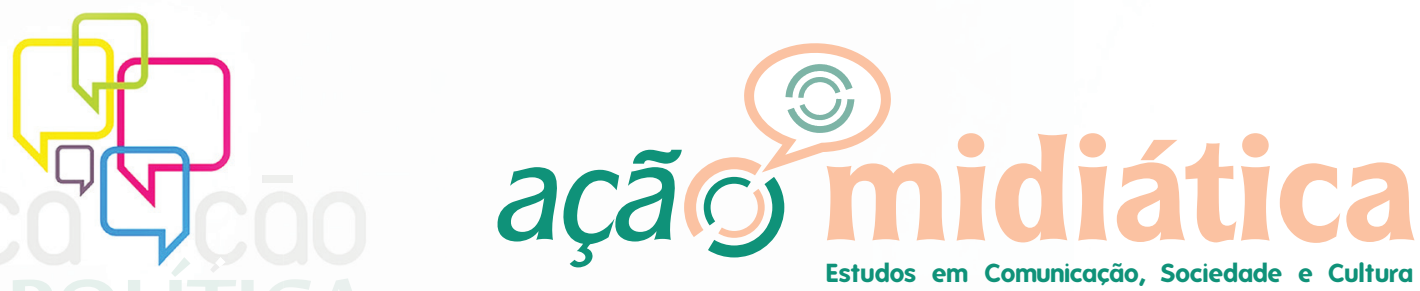

Estudos em Comunicação, Sociedade e Cultura

N. 6 | Ano 2013

Universidade Federal do Paraná | Programa de Pós-Graduação em Comunicação

\title{
La video vigilancia en el discurso modernizador de la seguridad ${ }^{1}$
}

Video vigilância no discurso de modernização da segurança

Video surveillance in the security modernizing discourse

Dolly Espinola FRAUSTO²

\begin{abstract}
RESUMEN
El desarrollo, producción e incorporación tecnológica constituyen un factor central en el desarrollo de las políticas de seguridad; el incremento acelerado de sistemas digitales para la tecnificación de la vigilancia representa un recurso que produce cambios sustanciales en la relación gobierno sociedad, de modo que el componente tecnológico adquiere una sobrevaloración de su eficacia tendiente a influir en la reducción de la percepción de la violencia y la mejora de la imagen de la acción de gobierno. La globalización del mercado y la consecuente transformación del espacio constituyen el punto de partida para observar el problema de los usos políticos y sociales de los sistemas de video vigilancia para el control del espacio público y el ejercicio del poder.
\end{abstract}

Palabras clave: Video vigilância; Control; Espacio público; Discurso político.

\section{RESUMO}

O desenvolvimento, a produção e a incorporação de tecnologia são um fator-chave no desenvolvimento das políticas de segurança; o rápido aumento de sistemas digitais para automação de vigilância representa um recurso que produz mudanças substanciais na relação entre o governo e a sociedade, de modo que a componente tecnológica torna-se supervalorizada na sua eficácia destinada a influenciar a redução da percepção de violência e melhorar a imagem da ação do governo. A globalização dos mercados e a consequente transformação do espaço constituem o ponto de partida para analisar o problema dos usos políticos e sociais dos sistemas de vídeo vigilância para o controle do espaço público e do exercício do poder.

Palavras-chave: Vigilância de vídeo; Controle; Espaço público; Discurso político.

\begin{abstract}
The development, production and incorporation of technology are a key factor in the development of security policies, the rapid increase of digital systems for the automation of surveillance, represents a resource that produces substantial changes in the relationship between government and society, so that the technological component becomes overvalued regarding its effectiveness at influencing the perception of violence and improving the image of the government action. Market globalization and the consequent transformation of space constitute the starting point to look at the problem of political and social uses of video surveillance systems for the control of public space and the exercise of power.
\end{abstract}

Keywords: Video surveillance; Control; Public space; Political discourse.

1 Trabalho apresentado à sexta edição da Revista Ação Midiática - Estudos em Comunicação, Sociedade e Cultura, publicação ligada ao Programa de Pós-Graduação em Comunicação, da Universidade Federal do Paraná.

2 Profesor-investigador Departamento de Educación y Comuncación. Universidad Autónoma Metropolitana. dolly.espinolaf@gmail.com 


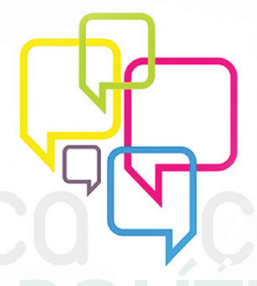

ação mididiática

Estudos em Comunicação, Sociedade e Cultura
N. 6 | Ano 2013

Universidade Federal do Paraná | Programa de Pós-Graduação em Comunicação

\section{Introducción}

Diversos enfoques de análisis dan cuenta del impacto que las tecnologías de información y comunicación tienen (TIC) en prácticamente en todos los ámbitos de la vida social; desde nuestra perspectiva, la atención se centra en la emergencia de nuevas formas de interacción que producen alteraciones sustanciales en el ejercicio del poder político resultado de la creciente incorporación de sistemas digitales para la tecnificación de la seguridad. En particular, interesa observar que el creciente desarrollo de los sistemas de video vigilancia como factor clave en la prevención, investigación e impartición de justicia tiene un efecto mayor a nivel de la percepción sobre la seguridad que sobre la reducción de los índices delictivos. Observar que este proceso se da sobre todo como resultado de las estrategias de comunicación que el poder político despliega a favor de las decisiones de gobierno, nos permite entender que no es posible establecer una relación directa entre la tecnificación y la mejora de las condiciones de seguridad que prevalecen en contextos determinados.

Si consideramos que el contexto Latinoamericano está marcado por profundas diferencias socio-culturales, resulta de importante profundizar en la reflexión sobre los usos sociales y políticos de las tecnologías digitales que no escapan a lógica de las estratégias discursivas del comercial y de mercado que las caracteriza y les confiere un aura de objetividad y eficacia en su incorporación a diversos procesos de registro, organización y sistematización de información y donde, en no pocas ocasiones, la racionalidad tecnológica se impone a la racionalidad humanista y deja de lado el diferencial socio-cultural que les da sentido.

Por lo anterior, interesa particularmente poner atención en el problema del uso de los sistemas de video vigilancia como resultado del creciente desarrollo de los sistemas digitales para la producción, sistematización y difusión de información que, en diversos ámbitos, forma parte del proceso de toma de decisiones en la definición de las políticas de seguridad. Si bien el problema del control y la vigilancia social no es un asunto nuevo, el desarrollo y expansión creciente de la video vigilancia representa un problema teórico y de análisis empírico que requiere de aproximaciones en sus dimensiones y aspectos éticos, legales y de políticas públicas. (ARTEAGA, 2009).

Frente a la amenaza permanente en que viven las megaciudades - actos terroristas, crimen organizado vinculado al narcotráfico, infiltración y debilitamiento de las instituciones del Estado - el diseño de nuevas formas de gestión de la seguridad en sus niveles global, regional y local tiene como una de sus consecuencias más evidentes el desdibujamiento de las fronteras entre lo público y lo privado.

Así, el diseño de políticas públicas que reconfiguran la relación entre la sociedad y las estructuras del poder político mediante el uso de dispositivos técnicos que extienden la capacidad de "ver", es decir, que incrementan el margen de certidumbre sobre el control de un 


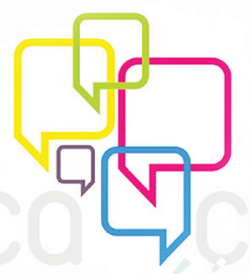

ação midiáática

N. 6 | Ano 2013

Universidade Federal do Paraná | Programa de Pós-Graduação em Comunicação

espacio determinado, tiene implicaciones en el ejercicio de derechos ciudadanos fundamentales como el de la privacidad.

La vigilancia y el control que se ejerce como resultado de múltiples dispositivos técnicos de registro de la realidad, adquieren un valor clave en el ejercicio de poder. Como lo describe Mattelart, los métodos de registro desplegados para mantener el control, o todos los dispositivos utilizados para vigilar actúan a favor de la gestión del poder y de su reproducción.

\begin{abstract}
En el plano de las mentalidades colectivas, se ha creado una suerte de habituación que ha ampliado los umbrales de la tolerancia y ha hecho que muchos consientan, muchas veces sin siquiera darse cuenta, importantes abandonos de su esfera privada y de sus derechos fundamentales. Y ello no sólo en relación con las técnicas de vigilancia y fichado, sino también de los instrumentos de medida y captación de las vivencias individuales por parte del complejo mediático y publicitario. En el seno mismo de los sectores democráticos y progresistas, sigue siendo fuerte la imposición de visiones instrumentalistas de la información, la comunicación y la cultura. (MATTELART, 2009, p. 252).
\end{abstract}

Esta creciente tolerancia a la exposición de la vida privada y la necesidad de contar con dispositivos que amplían nuestras capacidades y operan bajo el principio del domínio sobre el entorno, hace posible que los sistemas de video vigilancia al reconfigurar la esfera de lo público conviertan al conjunto de la sociedad en sujeto de sospecha permanente $\mathrm{y}$, al mismo tiempo, en sujeto vigilante. Si bien con los sistemas de control de que disponemos no es posible asegurar mejores formas de seguridad si se da una reconfiguración del ejercicio del poder y la resignificación el espacio público como el sitio de la desconfianza.

Los atentados terroristas del 2001 en los Estados Unidos dieron lugar a fuertes modificaciones en las políticas vinculadas a la protección y resguardo de la seguridad nacional. La idea de que el Estado norteamericano estaba perdiendo el control, incluso sobre su propio espacio aéreo y que se encontraba vulnerable y en amenaza permanente, dio lugar al diseño de estrategias y mecanismos que devolvieran la certidumbre ante la prueba evidente de la incapacidad de brindar protección y seguridad a la población civil.

Bajo esa perspectiva, la expansión de los sistemas de video vigilancia se construye en el discurso como una opción moderna que mejora la capacidad de control y de la gestión del poder político para disuadir y castigar cualquier amenaza, lo que produce y refuerza la idea de que así se alcanza una valoración positiva sobre la acción de gobierno por parte de los ciudadanos. Y al mismo tiempo, al utilizar los argumentos de la neutralidad y eficacia tecnológica se genera un proceso de enmascaramiento sobre el debilitamiento y fragilidad del orden institucional.

Por lo anterior, es necesario considerar que ni la perspectiva de la conspiración, ni la eficacia tecnológica resultan suficientes para atender de forma integral los problemas de la 

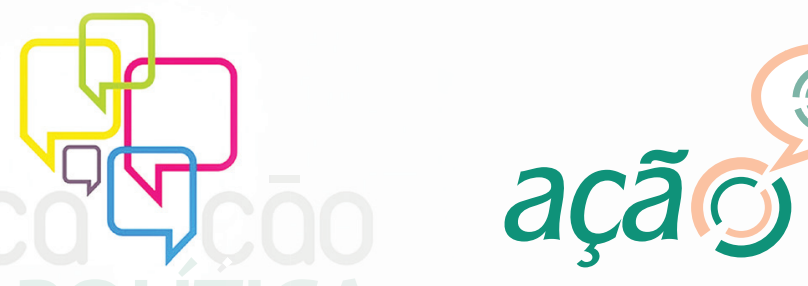

Estudos em Comunicação, Sociedade e Cultura

N. 6 | Ano 2013

Universidade Federal do Paraná | Programa de Pós-Graduação em Comunicação

seguridad y desarrollar acciones que garanticen la equidad en el acceso a la TIC y el desarrollo de métodos alternativos para garantizar el bienestar de los ciudadanos (LYON, 2008).

El desarrollo de las tecnologías digitales aplicadas al diseño de sistemas de tecno vigilancia, requiere de toma de decisiones y tiene implicaciones a distintos niveles, entre otros:

- La elaboración de los criterios para establecer la cobertura geográfica de los sistemas.

- La definición del diseño institucional responsable de la organización y procesamiento y resguardo de la información que se registra.

- Definir los recursos y acordar los protocolos de acción de los cuerpos de seguridad del estado.

- Atender a los problemas que derivan del impacto social que tienen para ejercicio de derechos fundamentales.

- Definir las condiciones para el uso de la información que se registra como prueba jurídica.

Esto último, debe ser visto a la luz de las nuevas formas de interacción en el espacio virtual donde se registra y comparte información de forma consentida sobre nuestros movimientos, formas de consumo o preferencias políticas y que define nuevos tipos de relación entre ciudadanos e instituciones. Lo que algunos autores describen como el monitoreo o vigilancia lateral (ANDREJEVIC, 2007) y que, como se ha señalado antes, supone de manera implícita o explícita la tolerancia cada vez mayor a la mirada y escrutínio público.

Entender cómo el uso de la video vigilancia en el contexto de violencia que predomina en el país, se inscribe en el marco del desarrollo del proyecto político económico que, a partir de la década de 1990, privilegia en México el modelo neoliberal y que da como resultado el desarrollo de políticas que favorecen el crecimiento de los sistemas de video vigilancia como una estrategia que, al amparo de la eficacia tecnológica, permite enmascarar la incapacidad institucional para garantizar las condiciones de seguridad a las que tiene derecho la ciudadanía.

El escenario de violencia que se vive en México ha trastocado los límites del orden institucional a todos sus niveles lo que lleva al poder público a desarrollar políticas de seguridad que no contribuyen a atender la problemática en su origen y, en ocasiones, trastocan el orden social mediante la visibilización masiva de la violencia y la impunidad. En ese sentido, es necesario reiterar que los métodos de registro desplegados para mantener el control o para vigilar actúan a favor de la gestión del poder y su reproducción; y si bien los sistemas de control no aseguran mejores formas de seguridad, sí reconfiguran formas de ejercicio del poder (MATTELART, 2009).

Los sistemas de video vigilancia como instrumento de registro forman parte del despliegue a gran escala de dispositivos técnicos que intervienen el espacio público y privado, y cuya expansión inicia a mediados del siglo pasado como resultado de políticas que, a nivel 


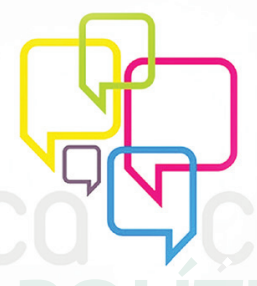

açãơ mididiática

N. 6 | Ano 2013

Universidade Federal do Paraná I Programa de Pós-Graduação em Comunicação

global, buscan asegurar la estabilidad geopolítica mediante el uso masivo de sistemas digitales para el registro y control social. Así, el uso político de las tecnologías de la imagen como instrumentos del control se justifican en el discurso de la seguridad bajo los argumentos de la eficacia tecnológica en la reducción de riesgo y la administración del espacio público.

En el escenario de expansión de los sistemas de video vigilancia, el diseño de nuevas formas de gestión de la seguridad supone cambios en las estructuras institucionales $\mathrm{y}$, en consecuencia, políticas públicas que generan procesos de transferencia creciente de responsabilidades sobre la seguridad hacia todos los niveles de la estructura social. Al respecto, resulta pertinente el señalamiento de Mattelart:

La multiplicación de las medidas de excepción en el marco de la "guerra global" contra el terrorismo se ha conjugado en el marco de los países democráticos a raíz del 11 de septiembre de 2001 con la creciente injerencia de los dispositivos de vigilancia en la vida cotidiana de los ciudadanos. A más seguridad, más tecnología: la cuestión de la seguridad del riesgo se va convirtiendo en una cuestión de técnicas (MATTELART, 2009, p. 11).

Desde este punto de vista, la seguridad deja de ser un problema de cuerpos especializados del estado para convertirse en un problema de vigilancia mutua entre los sujetos y su entorno donde los dispositivos y sistemas del video registro resultan un actor central a partir de la sobre valoración que se impone incluso al ejercicio de las libertades y que establece nuevos vínculos para el intercambio de información. De ahí que, como señala Lyon (2006) resulta necesario avanzar hacia una visión que a principios del siglo XXI, nos lleve hacia una mirada que esté más allá de los límites del panopticon, es decir, poner atención a las implicaciones que la video vigilancia tiene en el contexto en que ocurre.

Una aproximación general nos permite señalar que, la gestión del poder en el desarrollo de la modernidad ha supuesto múltiples formas de registro, almacenamiento y construcción de archivos que ofrecen a las estructuras políticas información privilegiada. Así, el control social se fue convirtiendo en un problema de diseño, registro y acceso a información que en el contexto institucional adquiere el carácter de evidencia judicial que, en el caso de la imagen, se da más por su capacidad para instalarse como verdad en el escenario social que como prueba sobre lo distinto y, por lo tanto, peligroso. Sin embargo, el problema de la objetividad de la imagen está siempre presente y aunque la representación visual ha sido objeto de manipulación sea sólo por la elección del punto de vista, o bien por la disposición que actualmente existe de gran cantidad de recursos digitales que permiten editar, trucar o recomponer el registro visual, la credibilidad le sigue siendo consustancial.

El incremento de los sistemas tecnológicos para el control de espacio público que se ha dado en última década en México, especialmente en la Ciudad de México, tiene implicaciones 

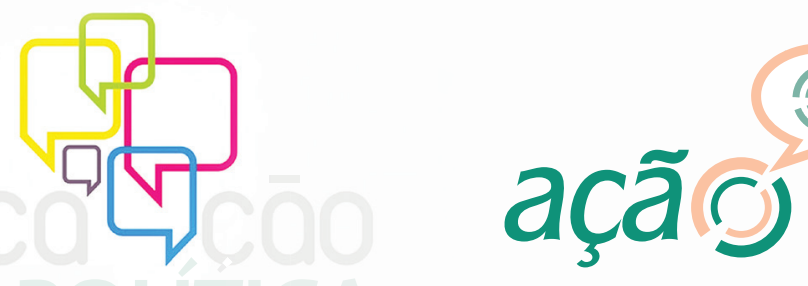

Estudos em Comunicação, Sociedade e Cultura

N. 6 | Ano 2013

Universidade Federal do Paraná | Programa de Pós-Graduação em Comunicação

de orden económico, político y social que requieren especial atención. Por un lado, las políticas de seguridad del gobierno de la Ciudad definen como una prioridad la adquisición de equipo de alta tecnología para garantizar la seguridad de la población y, por otro, la percepción de la violencia en la Ciudad de México se encuentra entre las más altas del país.

En la década pasada, el incremento de la violencia asociada al crimen organizado dio origen a que, en diciembre de 2006, el Presidente Felipe Calderón hiciera la declaración de la guerra contra el narcotráfico. La crisis de seguridad produjo que en 2009, The failed States Index 2012 publicado por la revista Foreign Policy colocó a México en el lugar 98 de 177 de las naciones con menor estabilidad en el mundo.

\section{La video vigilancia y la experiencia de la seguridad}

La amenaza del terrorismo a nivel global ha sido uno de los factores fundamentales para la expansión a gran escala de la tecno vigilancia, es un hecho que en los países desarrollados ese crecimiento se asocia a políticas de seguridad nacional que caracterizan la organización geopolítica a partir de la segunda mitad del siglo XX. (ARTEAGA, 2009). La tecno vigilancia aparece como respuesta a la amenaza global y tiene un desarrollo desigual que se inscribe tanto en el diseño de las políticas de seguridad como en la disposición de los recursos necesarios para su adquisición, operación y mantenimiento.

Algunos reportes señalan que el Reino Unido es el país con mayor número de cámaras instaladas con más de cuatro millones. (WAITON, 2010, p. 68) No obstante, un informe presentado por el Partido Liberal en el año 2007 sobre la efectividad del uso de cámaras, indica que no existe evidencia de que a más cámaras el índice criminal disminuye.

Un reporte de la New York Civil Liberties Union, del 2006, indica que si bien, la tasa de criminalidad descendió en 1997 cuando se puso en marcha el programa VIPER, este descenso no está directamente vinculado a la instalación de las cámaras, sino a la implementación de un sistema computarizado a principios de la década que permitió un uso más efectivo de los recursos policíacos.

El reporte también señala que los derechos de privacidad, asociación y libertad de expresión se ven comprometidos y que esto se debe a la posibilidad de convertir a los individuos en objetivos determinados a partir de características físicas o comportamientos sociales. En consecuencia, ciertos individuos son más susceptibles de convertirse en potenciales transgresores del orden social y advierte sobre la necesidad de contar con una legislación que reconozca que la video vigilancia afecta derechos y libertades fundamentales por que es necesario un balance entre el interés de protección a la seguridad pública y la privacidad individual.

En España, el número de cámaras en ese país se triplicó entre 2007 y 2008, pasando de 5 mil a 15 mil; y para el año 2010, la cifra creció hasta las 21 mil cámaras. Es importante 

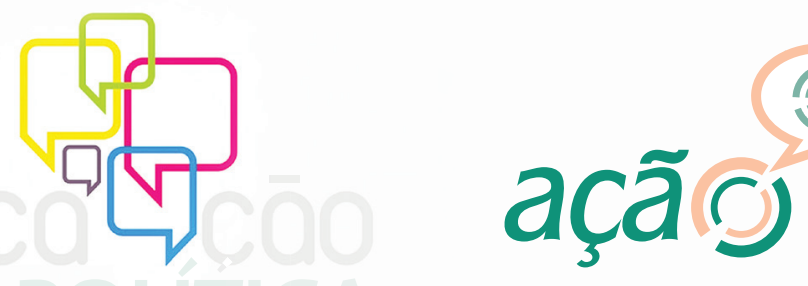

Estudos em Comunicação, Sociedade e Cultura

N. 6 | Ano 2013

Universidade Federal do Paraná | Programa de Pós-Graduação em Comunicação

señalar que España cuenta con un sistema legal que regula su uso a fin de proteger y garantizar el derecho de los sujetos a conservar su derecho a la privacidad. En 2006, la Agencia Española de Protección de Datos (AEPD), emitió una resolución relativa al tratamiento de los datos personales con fines de vigilancia a través de sistemas de video vigilancia.

Un estudio realizado por la Universidad de Málaga para evaluar la regulación jurídica y eficacia del sistema de video vigilancia instalado en el centro histórico de esa ciudad, indica que no existe suficiente investigación empírica sobre el tema pero que, al menos en el período de evaluación 2006-2008, no se pudo concluir que se haya disminuido significativamente el índice delictivo y que existe un fenómeno de desplazamiento hacia las llamadas zonas ciegas (DIÉZ-RIPOLLÉS y CEREZO, 2009).

Quizá debido al incremento acelerado de los sistemas de video vigilancia en um periodo tan corto, las reacciones en el barrio de Lavapiés en Madrid, España, habían provocado una situación conflictiva entre los ciudadanos y el gobierno. Para el año 2010, estas reacciones incluyeron asambleas, pintas en las calles y un concurso de carteles en contra de su instalación. Algunos resultados de ese movimiento dieron cuenta de una acción concertada de grupos que, mediante la participación en redes sociales y la producción y difusión de imágenes en contra de la instalación de las cámaras lograron contener lo que se consideró como una amenaza al derecho a la imagen y la privacidad.

Un estudio realizado por Sara Löfberg (2009) sobre el sistema denominado "ojos de águila" que inició su operación en 2002 con ocho cámaras instaladas en distintas zonas de Quito, Ecuador, también señala que no fue posible establecer decrementos significativos en la comisión de delitos y se apunta hacia las dificultades que ocasiona no contar con estrategias integrales de atención a la seguridad de los ciudadanos. Como ocurre con la evaluación realizada en la Málaga, los resultados también dan un desplazamiento de la violencia hacia las zonas ciegas. En el caso de Brasil, sabemos que el crecimiento del mercado de la video vigilancia está teniendo un crecimiento importante debido a la necesidad impuesta por la organización de los eventos deportivos del año 2014.

En México, un estudio realizado en 2006 en Huixquilucan, Estado de México, señala que: “...el sistema de videocámaras resultará en gran medida un producto orientado más al control social de ciertos sectores. Cuando fue posible percibir su utilización para el cuidado, respondía más que nada a los requerimientos de los sectores sociales mejor posicionados en la jerarquía social del municipio" (ARTEAGA, 2006, p. 50)

En la Ciudad de México, el desarrollo del sistema fue una pieza clave en las políticas de seguridad del gobierno de Marcelo Ebrard (2006-2012). La instalación de los Centros de Comando y Control y el aumento de cámaras de seguridad aparecen como las acciones de mayor importancia en su último informe de actividades. El tema de la modernización de la 

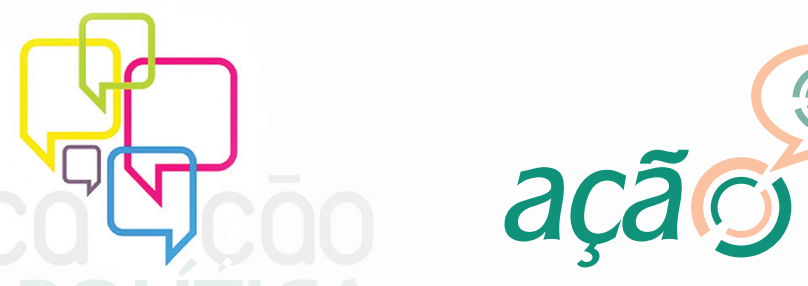

Estudos em Comunicação, Sociedade e Cultura

N. 6 | Ano 2013

Universidade Federal do Paraná | Programa de Pós-Graduação em Comunicação

seguridad fue tan importante en la agenda de trabajo que representó uno de los factores que contribuyeron a que dos meses antes del término de su gestión fuera nombrado en septiembre de 2012 Presidente de la Global Network of Safer Cities.

Aún y cuando los resultados de la Encuesta Nacional sobre Inseguridad 2010, indican que en la Ciudad de México se percibe un contexto más violento de lo que las cifras de criminalidad señalan, la respuesta en el despliegue tecnológico ha cobrado gran importancia para el gobierno local y se coloca con toda claridad en el marco del discurso modernizador que ha caracterizado a las administraciones locales de izquierda que desde el año 1997 gobiernan la ciudad.

La expansión e intensificación del uso de las tecnologías digitales en el discurso del gobierno aparece desde hace más de una década como un factor importante para el desarrollo en sectores como el educativo y, por supuesto, el de la seguridad. La instalación del sistema de cámaras de video vigilancia que se lleva a cabo en el marco de los programas Ciudad Segura y Bicentenario de Seguridad, reportó al final del 2012 la existencia de más de 8 mil cámaras. En cuanto a la inversión económica para la adquisición del sistema, la licitación pública por 459,8 millones de dólares: "la ganó la alianza corporativa de Telmex y Thales Group" (GAYTÁN, 2010, p. 21). A lo anterior, se suman las más de cinco mil cámaras que se ubican en el Sistema de Transporte Colectivo Metro de la ciudad. Cabe destacar que Telmex, propiedad del empresario mexicano Carlos Slim, bien conocido por encabezar la lista de los hombres más ricos del mundo, en el año 2000 creó la Fundación del Centro Histórico de la Ciudad de México que, de acuerdo con la propia página del empresario, tiene como objetivo rescatar y revitalizar esta zona de la ciudad.

La gestión de la seguridad en el país ha tenido entre una de sus consecuencias más importantes el crecimiento de la industria especialmente durante la última década. Al respecto, algunos estudios señalan que de las más de diez mil empresas dedicadas a la seguridad que en el año 2004 operaban en el país, más del 20 por ciento se ubicaron en la Ciudad de México (WONDRATSCHKE, 2005, p. 9). Aunque resulta difícil señalar una correlación directa entre las cifras y el crecimiento de los sistemas de video vigilancia, lo cierto es que esto se explica en primer lugar por el desbordamiento de la problemática de la seguridad que afecta el orden institucional. No obstante, es necesario señalar que la organización del sistema político federalizado del país, se caracteriza en su funcionamiento por la falta de coordinación entre los distintos órdenes de gobierno, la proliferación de cuerpos de seguridad y la consecuente ineficacia en el desarrollo de políticas públicas suficientes para contender con la problemática existente.

En este contexto, los recursos públicos destinados al desarrollo de los sistemas de video vigilancia dependen directamente del orden de prioridades establecido en cada administración, lo que permite que el destino del gasto gubernamental tenga un componente importante en 
